International Journal of Biomedical Investigation

Available Online: http://ijbi.edwiserinternational.com

\title{
Analysis of Gene Expression and Neuronal Phenotype in Neuroscreen-1 (NS-1) Cells
}

\author{
Smritee Pokharel ${ }^{1}$, Chang Hun Lee ${ }^{2}$, Nailya Gilyazova ${ }^{1}$ and Gordon C. Ibeanu ${ }^{1,3 *}$ \\ ${ }^{1}$ Biomanufacturing Research Institute and Technology Enterprise (BRITE), North Carolina Central University, Durham, North \\ Carolina, United States of America \\ ${ }^{2}$ School of Dentistry, Medical College of Virginia, Richmond, Virginia, United States of America \\ ${ }^{3}$ Department of Pharmaceutical Science, North Carolina Central University, Durham, North Carolina United States of America
}

\section{Article info}

Received 28 November 2018

Revised 12 December 2018

Published 17 December 2018

*Corresponding author: Gordon C.

Ibeanu, Department of Pharmaceutical Science, North Carolina Central University, Durham, North Carolina, USA, Tel: +1-919-530-6711; E-mail: gibeanu@nccu.edu

\begin{abstract}
Neuroscreen-1 (NS-1) a sub-clone of pheochromocytoma (PC12) cell is gaining broad acceptance as in vitro neuronal model for biochemical and phenotypic assays due to robust growth and differentiation profiles. However, the molecular characteristics of the cell remains to be documented. In this study, we performed comparative analysis for expression of neuronal marker genes in undifferentiated and nerve growth factor (NGF) differentiated NS-1 and PC12 by qPCR and immunoblot assays. We show that differentiation of NS-1 occurred under low concentrations of NGF relative to PC12. Cell growth also occurred more rapidly in NS-1. Transcriptional analysis of neuronal marker genes showed comparable expression of tyrosine receptor kinases (Ntrk1, Ntrk2, NGFR/p75NTR) and muscarinic acetylcholine (Chrm1, Chrm2, Chrm3, Chrm4) receptors in unspecialized cells. Ntrk2, adenosine receptors (Adora1, Adora2A) and choline acetyltransferase (ChAT) were altered in undifferentiated NS-1. In contrast, Ntrk1, Ntrk2, Chrm2 transcripts were vastly increased in NS-1 with NGF exposure, while Ntrk3, Adoral and Adora $2 A$ transcripts were reduced. In differentiated PC12, Chrm 4 and ChAT were markedly upregulated. Our data suggests that differences in morphological and phenotypic characteristics that distinguish NS-1 from PC12 is likely the product of altered gene expression. Furthermore, expression of neuron type genes in NS-1 support its use as an alternative model to PC12.
\end{abstract}

Keywords: Neuroscreen-1; Pheochromocytoma; NS-1; PC12; Neuronal; Neurite; Transcription; Cholinergic; Differentiation; Nerve Growth Factor

\section{Introduction}

Development of in vitro disease model that closely mimics in vivo condition has become significant with the emergence of new disease and pathological conditions. Basic research for neurodegenerative disease largely relies on cell line studies, which also act as the primary platform for drug screening in translational medicine. However, complex nutritional requirements, slow growth properties, and expression of cell-type specific markers limit the choice of cell lines used in research. Neuroscreen-1 (NS-1), is a sub-clone of PC12, a noradrenergic clonal line of adrenal pheochromocytoma that shows dopaminergic properties and are widely used in neurobiology, neurotoxicology, and drug discovery studies [1-5] . 
Differentiation of PC12 by NGF is well documented. NGF treatment of PC12 cell leads to cessation of cell division, induction of neurite outgrowth, and production of electrically excitable cells in culture, mimicking characteristics of sympathetic-like neurons [1-6]. In addition to the neuroprotective and neuro-restorative properties of NGF, dysregulation in NGF signaling has been positively correlated with neurodegenerative disease including Alzheimer's disease (AD) [7-8], epilepsy [9] and cancer [1012]. AD is characterized by death of forebrain cholinergic neurons that provides cholinergic innervations to cerebral cortex and hippocampus. Neuritic plaques comprising $\beta$-amyloid fibrils, dystrophic neuritis, reactive astrocytes, phagocytic cells and protein fragments derived from degenerating neurons are the typical feature of $\mathrm{AD}$. NGF regulates proliferation and differentiation of neuronal cells via activation of activation of tyrosine protein kinase (TrkA) receptor, and downstream signaling molecules that include Ras/MAP kinase cascade, IP3-dependent $\mathrm{Ca} 2+$ release, and PI3K/Akt pathways [13]. TrkA also enhance neuronal survival by silencing the tumor necrosis factor receptor family member $p 75^{\mathrm{NTR}}$ [14]. Furthermore, NGF increases expression of ChAT and VAChT, two cholinergic specific markers that are required for cholinergic neurotransmission [15-16]. ChAT enables the synthesis of acetylcholine (ACh) from acetyl-CoA and choline, whereas VAChT acts as a membrane transporter loading ACh into secretory vesicle and makes it available for secretion [17]. Decrease in ChAT and VAChT is suggested to play a role in the progression of AD [18-19].

Muscarinic acetyl choline receptors (Chrms) are members of the G-Protein coupled receptor family, expressed in both the central nervous system (CNS) and peripheral nervous system (PNS). Chrms comprise of five genes (Chrm1, Chrm2, Chrm3, Chrm4 and Chrm5) which specify five membrane receptor subtypes (M1M5) divided into two broad classes based on their coupling efficiency with $G$ proteins. Muscarinic receptors found in the cholinergic system signal through the binding of acetylcholine and are thought to be involved in learning, memory [20,21], motor and sensory modulation [22] and thus has been implicated in a wide variety of physiological processes in the CNS and diseases such as Alzheimer's disease and schizophrenia [23] Parkinson's disease [24] vascular dementia and depression [25]. Similar to muscarinic receptors, the adenosine receptors (AR) are GPCRs present in the CNS and mediate the actions of adenosine in the release of neurotransmitters and synaptic plasticity [26]. There are four members of the family,
$A_{1}, A_{2 A}, A_{2 B}$, and $A_{3}$. Adenosine $A_{1}, A_{2 A}$, and $A_{2 B}$ receptor mRNAs have been detected in PC12 variants. Adenosine $A_{1}$ and $A_{2 A}$ receptors are present in the microglia and neurons, have relatively high affinity for adenosine, and demonstrate opposing actions in the CNS. $A_{1}$ receptors are prevalent in the synaptic regions whereas $A_{2 A}$ receptor is localized in the striatum and olfactory bulb [27]. The $\mathrm{A}_{2 \mathrm{~A}}$ receptor has been shown to modulate NGF-induced neurite outgrowth (NOG) in PC12 and neuritogenesis in primary hippocampal neuron in association with translin-associated protein $\mathrm{X}$ (TRAX) [28].

NS-1 cell line has been used as a neuronal cell model to study the chemical dependent initiation, progression, inhibition, and toxicity in neurite outgrowth assays $[2,5,29,30]$. Despite increasing interest in the use of NS1 as a substitute model for PC12, differences in growth and differentiation characteristic have not been studied at the molecular level. No comparative information exists in the literature on gene expression patterns between NS-1 and the parental PC12. Understanding traits of NS-1 gene expression in differentiated and naïve states is critical in defining the representative neuronal subtype for utility as a relevant substitute experimental model for PC12. In the present study, we characterized the gene and protein expression profiles by comparative analysis of neuronal molecular markers by qPCR and immunoblot analyses. We conclude that changes in gene and protein expression could account for enhanced phenotypic properties of NS-1, and that the expression of neuronal markers support the use of NS-1 as an alternative and substitute cell model to PC12.

\section{Materials and Methods}

Reagents were sourced from the following vendors: NS1 (Cellomics, Inc., Pittsburgh, PA), PC12 (ATCC, Manassas, VA), 2.5S murine NGF (Bachem Inc., Torrance, CA), RNA extraction kit and Turbo DNAfree kit and RETROscript Kit (Ambion Inc., Carlsbad, CA), RT PCR primers (Eurofins MWG Operon, Huntsville, AL); $\mathrm{RT}^{2}$ SYBR Green ROX qPCR Mastermix (QIAGEN, Valencia, CA), MTT (3-(4, 5dimethylthiazolyl-2)-2,5-diphenyltetrazolium bromide) (Sigma-Aldrich, St. Louis, MO), DMEM and RPMI 1640, HCS CellMask Red ${ }^{\mathrm{TM}}$, and Alexa Fluor 488 Donkey anti-rabbit antibody (Invitrogen, Carlsbad, CA), fetal bovine serum (FBS) (Hyclone, Logan, UT), heat inactivated horse serum (Lonza, Walkersville, MD), and L-glutamine and 1\% Penicillin/Streptomycin (BioWhittaker, Walkersville, MD), Mouse anti-ChAT and Rabbit anti-M2 antibody (EMD Millipore, Billerica, MA), Goat anti-VAChT (Promega, Madison, 
WI), Rabbit anti-TrkA (Cell Signaling, Tech., Danvers, MA), and Super Signal Pico Chemiluminescent Substrate (Thermo Fisher Sci., Grand Island, NY).

\section{Cell culture and differentiation}

PC-12 cells were maintained in collagen coated plates in DMEM containing 5\% FBS, $10 \%$ heat inactivated horse serum, $1 \%$ L-glutamine and $1 \%$ Penicillin/Streptomycin. NS-1 cells were maintained in RPMI-1640 supplemented with 10\% FBS, 1\% Lglutamine and $1 \%$ Penicillin/Streptomycin. The culture media was renewed every three days and cells subcultured at $80 \%$ confluence. Cell differentiation was accomplished in the presence of $20 \mathrm{ng} / \mathrm{mL}$ NGF in reduced serum media containing 2\% FBS. Following differentiation, cells were observed for change in morphology characterized by presence of elongated neurites. For dose response assays, NS-1 and PC-12 cells were treated with NGF in reduced serum media containing $0,3.13,6.25,12.5,25$ and $50 \mathrm{ng} / \mathrm{ml}$ of NGF respectively for $48 \mathrm{~h}$ followed by staining, capture of image and quantification of the neurite.

\section{Analysis of neurites}

The quantification of neurites was performed as described previously reported [5] with minor changes. Briefly, NS-1 cells were seeded in 384 well plate at a density of $5 \times 10^{2}$ cells per well and allowed to grow in the presence of $0,3.13,6.25,12.5,25$, and $50 \mathrm{ng} / \mathrm{mL}$ NGF respectively for $48 \mathrm{hrs}$. At neurite maturity, the differentiation media was aspirated from and replaced with $30 \mu \mathrm{L}$ of staining solution containing $0.2 \mu \mathrm{g} / \mathrm{ml}$ Hoechst dye and $0.25 \mu \mathrm{g} / \mathrm{ml}$ HCS CellMask Red ${ }^{\mathrm{TM}}$ (HCMR) in $4 \%$ formaldehyde. The plate was incubated in the dark at $23^{\circ} \mathrm{C}$ for 1 hour to obtain fixed samples with Hoechst-stained nuclei and HCMR-stained cell bodies and extensions. The staining solution was aspirated, cells rinsed with PBS, and images were acquired on a CellInsight ${ }^{\mathrm{TM}}$ CX5 High Content Screening (HCS) Platform. Images were captured by 10X objective in two different channels: Nuclei (Hoechst) with excitations of 386/23 nm, Cell body/ neurites (HCS Red) 650/13 nm. Neurites were analyzed using HCS Studio ${ }^{\mathrm{TM}}$ Cell Analysis Software- Neuronal Profiling v3.5 BioApplication (Thermo Fisher Scientific ${ }^{\mathrm{TM}}$ ).

\section{RNA isolation and RT-qPCR}

Total RNA isolated using Ambion RNA extraction kit was reverse transcribed with the RETROscript reverse transcription kit to obtain complementary DNA
(cDNA). Real time-qPCR was conducted with $2 \mu \mathrm{L}$ cDNA, $2 \mu \mathrm{L}$ of $10 \mu \mathrm{M}$ gene specific primer, $10 \mu \mathrm{L}(2 \mathrm{X})$ SYBR Green reagent and $6 \mu \mathrm{L}$ DNase/RNase free water. The amplification protocol entailed 40 cycles of denaturation at $94^{\circ} \mathrm{C}$ for $30 \mathrm{secs}$, annealing at 55 to $57^{\circ} \mathrm{C}$ for $30 \mathrm{sec}$ and extension at $72^{\circ} \mathrm{C}$ for $30 \mathrm{sec}$, followed by the final extension for $5 \mathrm{~min}$ at $72^{\circ} \mathrm{C}$ in a 7500 RT- PCR system (Applied Biosystems, Foster City, CA). Confirmation of specificity of PCR products was achieved by analysis of melting curves. Genes were analyzed and fold differences were calculated using RPL-19 as reference gene. The primers were designed by Primer 3 software (http://frodo.wi.mit.edu) using the sequence information listed at the National Centre for Biotechnology Information and sequence verification was done using BLAST. Detailed information on primer sequence is provided as a Table 1 .

\section{Immunoblot analyses}

Cells cultured in 6 well plates were washed in ice cold PBS and lysed in RIPA buffer containing protease inhibitor cocktail (ThermoFisher Scientific). Total protein was isolated and quantified by Bradford method [31]. A total of $20 \mu \mathrm{g}$ protein was reduced, electrophoresed in 10\% SDS-polyacrylamide gel and transferred to PVDF. Membranes were blocked, incubated with mouse anti-ChAT, goat anti-VAChT, or rabbit anti-TrkA, washed, incubated with species specific secondary antibody and visualized with Super Signal Pico Chemiluminescent Substrate (Thermo Scientific). Band intensity was measured densitometrically using ImageJ software and results were normalized with $\beta$-actin as internal control.

\section{Immunocytochemistry}

Cells allowed to grow and differentiate in glass slides in presence or absence of NGF for $24 \mathrm{~h}$ were stained as previously described [32]. Briefly, cells were rinsed with PBS, fixed in $4 \%$ formaldehyde, permeabilized in $0.2 \%$ Triton X-100. Cells were blocked and subsequently incubated with primary rabbit anti-M2 antibody overnight at $4^{\circ} \mathrm{C}$. Cells were further treated with secondary antibody (Alexa Fluor 488 Donkey antirabbit; Invitrogen, Eugene, OR, USA) followed by nuclei staining with mounting media containing 4', 6diamino 2-phenylindole (DAPI; Sigma-Aldrich). Cell pictures were taken using an Olympus inverted fluorescence microscope equipped with a digital camera (Nikon, Tokyo, Japan).

\section{Statistical analyses}


Statistical analyses were performed in GraphPad PRISM 7 (GraphPad, La Jolla, CA, USA). Data are provided as means \pm standard error (SEM) of at least three independent experiments. Significant differences between groups were determined using either multiple t-test or two-way ANOVA. A nominal $p$-value of less than 0.05 was considered to be statistically significant

\section{Results}

Analyses of NGF-dependent neurite outgrowth in differentiated cells

NS-1 is a clonal line of the pheochromocytoma cell PC12, which has the ability to differentiate into neuronal-like cells in presence of NGF. NS-1 and PC12 cells exhibit phenotypic and functional characteristics associated with NGF differentiation in altered timescales. To explore the underlying causes for these differences in characteristics we began by evaluating the NGF-dependent neurite growth dynamics in NS-1 and PC12 cells seeded overnight in growth media and treated with increasing concentrations of NGF. Morphological appearance of the cells was assessed visually and quantified for neurite length and branch points after $48 \mathrm{~h}$ in culture. NGF treatment induced hypertrophy in NS-1 and PC12 and produced cells with enhanced neuron-like morphological characteristics. NGF stimulation led to increase in cell volume, change in shape and appearance, and formation of neuritic processes (Figure 1A, upper panel).

Table 1. Sequences of primers used for PCR

\begin{tabular}{|c|l|l|c|}
\hline Name & \multicolumn{1}{|c|}{ Forward Sequence $\left(\mathbf{5}^{\prime} \rightarrow \mathbf{3}^{\prime}\right)$} & \multicolumn{1}{c|}{ Reverse Sequence $\left(\mathbf{5}^{\prime} \rightarrow \mathbf{3}^{\prime}\right)$} & Length \\
\hline Ntrk1 & AGCACAGACTACTACCGTGTGG & CACATCACTCTCGGTCTGAC & 22 \\
\hline Ntrk2 & TTCCAAGTTTGGCATGAAAGGC & CGAAGAAGACGGAGTGTTGCTC & 22 \\
\hline Ntrk3 & TGCCTGATGTGGACTGGATA & TGTCTTCGCTCGTCACATTC & 20 \\
\hline Chrml & AACATCACTGTCTTGGCACCAG & ACCAGTAGGTTGCCTGTCACTG & 22 \\
\hline Chrm 2 & ATACCCTCTACACTGTGATTGGC & TTCATAACGGAGGCATTGCTGAC & 23 \\
\hline Chrm3 & ATGCCTCTGTCATGAATCTGCTG & GCCAGACCAATCATCACACCAC & 23 \\
\hline Chrm4 & CCAGAGCACAAAGGACAAGACC & CCAGAGCACAAAGGACAAGACC & 22 \\
\hline ChAT & CTCCCCAAAAGATGCCTGTA & CTGGCTCTTCCTGAACTGCT & 20 \\
\hline NGFR & CACCGACAACCTCATTCCTGTC & TTGTTTGCAGCTGTTCCACCTC & 22 \\
\hline Adoral & AGATCCCTCTCCGGTACAAGAC & AGCCAAACATGGGTGTCAGG & 22 \\
\hline Adora2A & AGCCAGAGCAAGAGGTATCTCAG & CGAAGACGTTCTCACAGACGAC & 23 \\
\hline
\end{tabular}

Differentiated NS-1 developed dense neurite networks characterized by long neurites seen in the phase contrast micrograph and HCS Red stained image (Figure 1A, lower panel), and cell aggregates visible in PC12 were conspicuously absent in NS-1. Quantitative assessments of neurite lengths and branch points by progressively increasing the concentration of NGF confirmed a rapid dose-dependent response in neurite outgrowth, sustained neurite elongation, and increased neurite branch points in NS-1 at $48 \mathrm{~h}$ post NGF treatment. As little as $3.13 \mathrm{ng} / \mathrm{mL}$ of NGF was sufficient to induce quantifiable neurite outgrowth at $48 \mathrm{~h}$ in NS-1 cells, achieving a plateau at approximately $12.5 \mathrm{ng} / \mathrm{mL}$ NGF
(Figure 1B). The maximum neurite length of $\sim 8 \mu \mathrm{m}$ recorded for NS-1 was 8-times greater than the length of neurites in $\mathrm{PC} 12$ and the branch points in NS-1 were 10 -fold more than PC12. Although differentiation of PC12 resulted in neurite outgrowth the lengths did not change significantly with increasing concentrations of NGF up to $50 \mathrm{ng} / \mathrm{mL}$, and very few branch points were detected (Figure 1C). To evaluate the proliferative capacity, the growth profile of NS-1 and PC12 were measured at various time points up to $72 \mathrm{~h}$ in culture. We observed no significant differences in cell proliferation up to $35 \mathrm{~h}$ as measured by cell viability assay (Figure 1D). However, clear divergence in cell 
proliferation was seen beginning at $\sim 40 \mathrm{~h}$ in NS-1 relative to $\mathrm{PC} 12$, culminating in a 2 -fold difference in fluorescence intensity at $72 \mathrm{~h}$. The results of these experiments clearly demonstrate an increased sensitivity of NS-1 to NGF as observed by accelerated formation of neurites, neurite elongation, increased neurite networks, and enhanced proliferation compared to $\mathrm{PC} 12$.

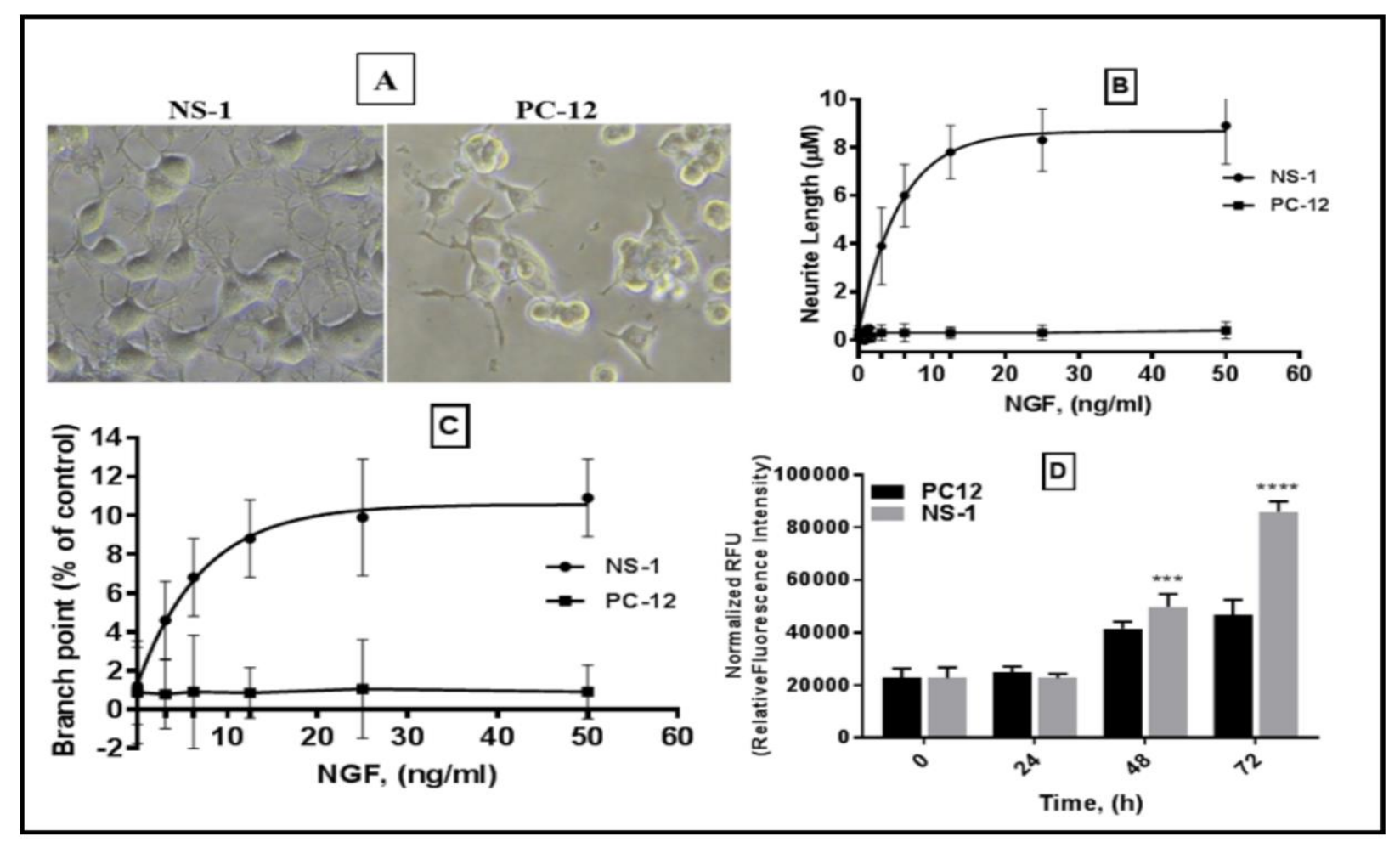

Figure 1: Morphometric analysis of NGF induced neurite outgrowth in NS-1 and PC12. Cells were treated with increasing concentrations of NGF for 48 h processes with HCS Red ${ }^{T M}$ and Propidium Iodide and analyzed for neurite length and branch points on the Cellomics imaging platform. (A) Phase contrast photomicrographs of NS-1 cells (top left panel) and PC12 (top right panel) treated with $50 \mathrm{ng} / \mathrm{mL} \mathrm{NGF}$ showing presence of neurites and branch points. $(B)$ Graphical quantification of NGF concentration-dependent stimulation of neurite lengths (top) and branch points (bottom) in NS-1 and PC12. (C) Graph of growth analysis of NS-1 and PC12 cells acquired at various time points after inoculation in media. $(* * * p<0.001 ; * * * * p<0.0001$ indicates significance).

Transcriptional analysis of neuronal markers in naïve and NGF differentiated cells

To ascertain the identity relationships of NS-1 and PC12 cell lines, transcriptional analyses of gene profiles in undifferentiated and NGF differentiated cells were assessed by RT-qPCR. Genes that establish the identity of neuronal cells including tyrosine kinase (Ntrk) receptors, muscarinic receptors and cholinergic receptors were analyzed (Figure 2). The Ntrk2 transcript was reduced $\sim 2$-fold in undifferentiated NS-1 compared to PC12 cells. In contrast, when cells were differentiated with NGF, the transcription of Ntrk1 and Ntrk2 were highly upregulated in NS-1 by $\sim 2.5$-fold and $\sim 1.5$-fold respectively, while Ntrk3 was significantly decreased compared to untreated cells (Figure 2B). No change occurred in the transcription of Ntrk genes in differentiated or undifferentiated PC12. When expression of muscarinic receptors was quantified, we observed no statistically significant differences in any Chrm gene profile between the two cell lines in undifferentiated state (Figure 2C). After treatment with NGF, differentiation of the cells was accompanied by significant upregulation of Chrm2 alone in both cells with NS-1 expressing $\sim 2$-fold $(\mathrm{p}<0.01)$ increase of Chrm 2 while PC12 showed a modest but significant increase in transcription of the Chrm2 (Figure 2D). In contrast, the transcription of Chrm4 revealed a highly dissimilar pattern of regulation between NGF-treated cells. Chrm 4 increased $\sim 3$-fold $(\mathrm{p}<0.001)$ compared to unexposed cells, whereas, no change in gene transcription was observed in NS-1. Interestingly, the increase of Chrm4 detected in undifferentiated NS1 was significantly inhibited by NGF treatment relative to background expression of untreated PC12 cells. 
Citation: Pokharel S, Lee CH, Gilyasova N, et al. Analysis of Gene Expression and Neuronal Phenotype in Neuroscreen-1 (NS-1) Cells. Int J Biomed Investig 2018; 1: 115. doi: 10.31531/2581-4745.1000115
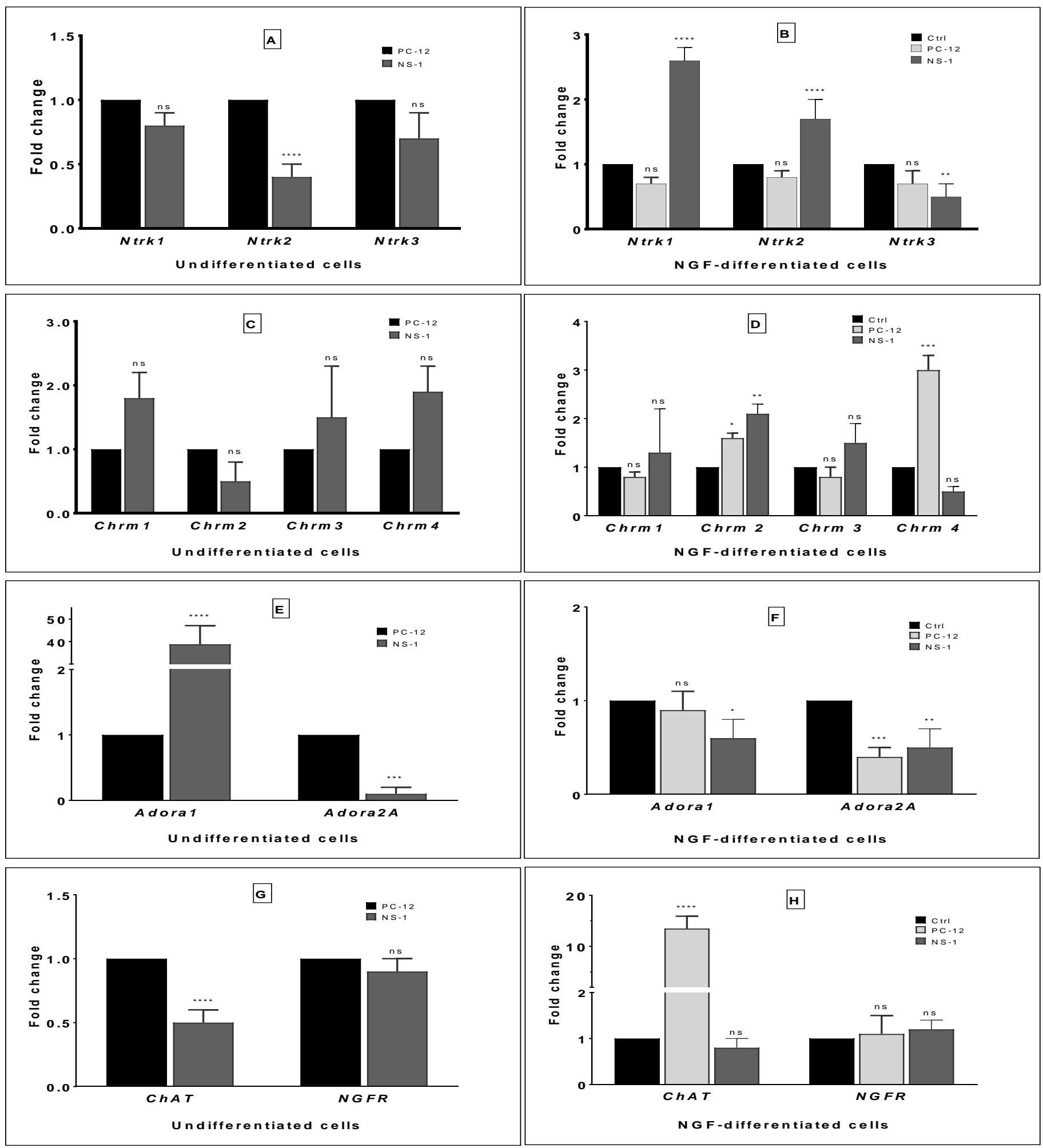

Figure 2: Analysis of transcription patterns of neuronal genes. Transcription of the neuronal marker genes Ntrk, Chrm, Adora, Chat, and Ngfr were measured in naïve and NGF exposed NS-1 and PC12 cells. Fold change in gene transcripts are shown as bar graphs in (A) undifferentiated and (B) NGF-differentiated Ntrk genes (Ntrk1, Ntrk2, and Ntrk3); (C) undifferentiated and (D) NGF-differentiated Chrm genes (Chrm1, Chrm2, Chrm3, Chrm4); (E) undifferentiated and $(F)$ NGF-differentiated Adora genes (Adoral, Adora2A); (G) undifferentiated and $(H) N G F$ differentiated ChAT and Ngfr genes. The data shows the means $\pm S D$ of at least three biological replicates $\left({ }^{*} p<0.05\right.$; $* * p<0.01 ; * * * p<0.001 ; * * * * p<0.0001$; indicates relative significance. $n s=$ not significant $)$. 
Analysis of Ntrk genes in undifferentiated cells revealed no statistical difference ( $\mathrm{p}>0.05)$ in Ntrk1 and Ntrk3 but showed a significant difference in transcriptional regulation of Ntrk2 ( $<<0.001)$ (Figure 2A).

Analysis of adenosine gene transcripts showed high a degree of difference in the level of transcripts of two receptor subtypes analyzed in NS-1. A 40-fold ( $\mathrm{p}<0.0001)$ increase in Adoral mRNA was detected in NS-1 untreated with NGF relative to PC12, while Adora $2 A$ transcript was barely detectable in the same cell line (Figure 2E). NGF-treatment was accompanied by a massive reduction in the magnitude of Adoral transcript in NS-1 to nearly one-half that of untreated control with no alteration in transcript level in PC12 (Figure 2F). While Adora2A transcript was undetectable in naïve NS-1, NGF exposure led to an increase in transcript of $\sim 50 \% \quad(\mathrm{p}<0.01)$ of untreated control. Interestingly, differentiation of PC12 with NGF resulted in significant reduction in Adora2 mRNA, although the transcript levels in both cell lines were significantly less than undifferentiated cells.

Finally, we assessed the transcript abundance of two cholinergic markers, ChAT, and the low affinity neurotrophin receptor NGFR $/ p 75^{N T R}$. In control cells not exposed to NGF, the transcript level of ChAT was significantly less in NS-1 at $\sim 50 \%(\mathrm{p}<0.0001)$ the magnitude in PC12 (Figure 2G). However, NGF differentiation led to $\sim 14$-fold $(\mathrm{p}<0.0001)$ increase of ChAT gene activity in PC12 but had modest effect in NS-1 (Figure 2H). NGFR gene activity as neither altered in undifferentiated nor differentiated forms of NS-1 and PC12.

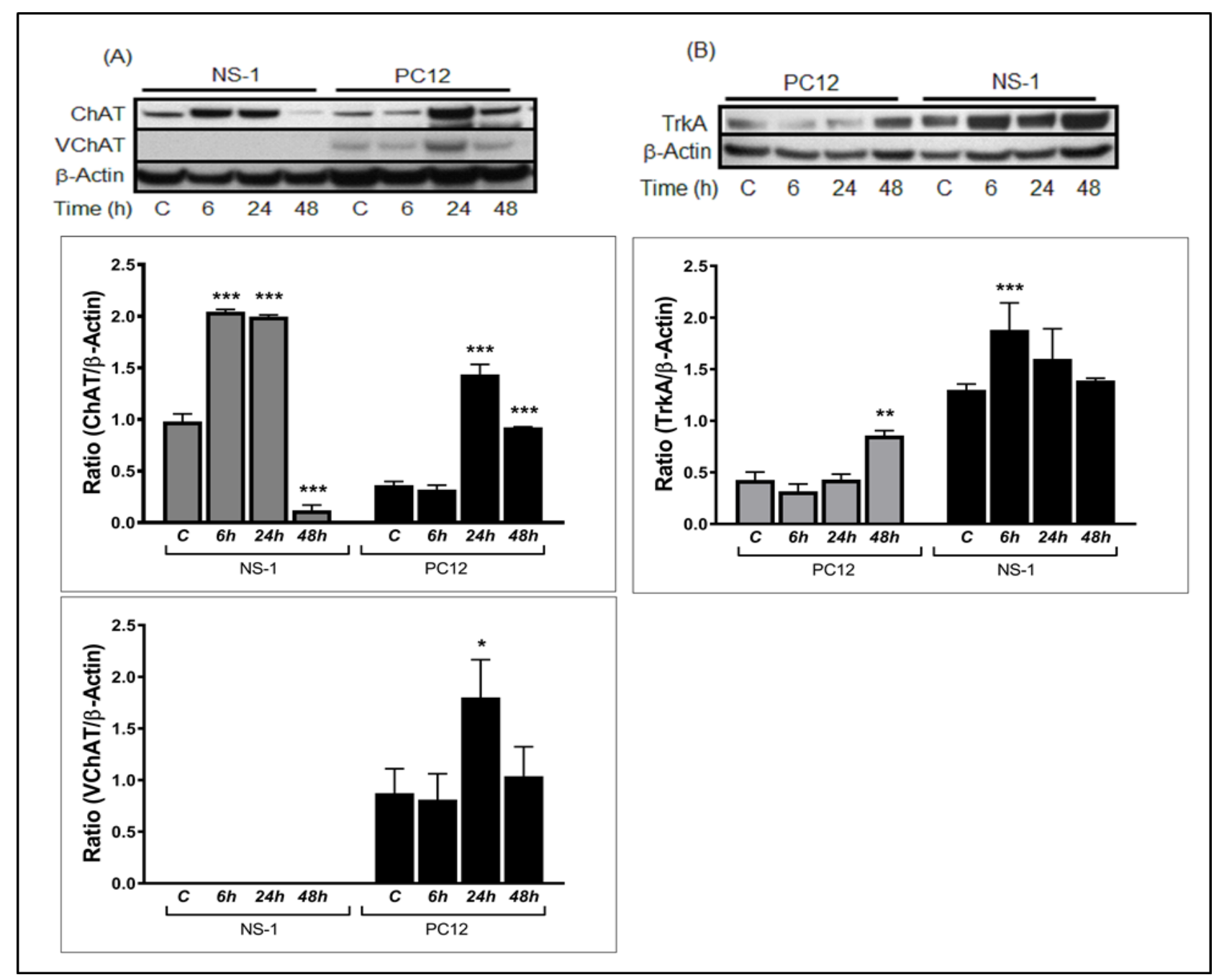

Figure 3: Evaluation of neuronal protein expression in differentiated NS-1 and PC12 cells by immunoblot analyses. Cells were treated with NGF for $6 \mathrm{~h}, 12 \mathrm{~h}$, and $24 \mathrm{~h}$ and the cellular lysates subjected to western blotting with specific antibodies to (A) ChAT and VAChT, and (B) TrkA proteins. Quantification of the relative expression of ChAT, $V A C h T$, and TrkA is provided in the bar graphs. $\beta$-actin was used as an internal control of protein expression. $\left({ }^{*}<<0.05\right.$ and ${ }^{* * *} p<0.001$ indicates significance compared with untreated controls labeled $C$ in the figure). 


\section{Immunoblot analysis for expression of cholinergic markers in NS-1 and PC12 cell lines}

Our investigation of transcription of neuronal indicators showed significant changes in gene transcription patterns of naïve and NGF-differentiated cells in NS-1 and parental PC12 cell lines. To verify whether changes observed in gene transcription were translated to protein expression, we measured the amount of proteins in both naïve and NGF differentiated cells by immunoblot. The molecules analyzed included ChAT, VAChT, and TrkA (Figure 3).

We found that NS-1 and PC12 cells both showed detectable expression of ChAT in undifferentiated state. However, the basal expression of ChAT protein in NS1 was markedly higher than the basal expression in PC12 (Figure 3A). NGF treatment of NS-1 led to 2-fold $(\mathrm{p}<0.001)$ elevation in ChAT expression within $6 \mathrm{~h}$ in culture and the amount of protein detected in the cell remained unaltered at $24 \mathrm{~h}$ post NGF treatment, then subsequently decreased 9-fold $(\mathrm{p}<0.001)$ at $48 \mathrm{~h}$ compared control cells. Likewise, differentiated PC12 showed significant 4-fold $(\mathrm{p}<0.001)$ increase of ChAT expression relative to untreated $\mathrm{PC} 12$ but at protracted time point $(24 \mathrm{~h})$ relative to upregulation in NS-1 which occurred in $6 \mathrm{~h}$. Unlike NS-1 which showed nearly undetectable ChAT protein at $48 \mathrm{~h}$, the level of ChAT in NGF-differentiated PC12 at $48 \mathrm{~h}$ decreased to a certain extent but was very robust at 2.6 -fold $(\mathrm{p}<0.001)$ relative to the control $\mathrm{PC} 12$.

Meanwhile analysis of the degree of expression of VAChT showed that the protein was not expressed in undifferentiated or NGF differentiated NS-1 cell (Figure 3A). In contrast, VAChT protein was detected in untreated $\mathrm{PC12}$, although in minute quantity. Similarly, the amount of VAChT detected in PC12 cell subjected to NGF treatment for $6 \mathrm{~h}$ was not significantly different to baseline expression seen in control cells. However, at $24 \mathrm{~h}$ post NGF differentiation, the expression of VAChT was significantly elevated by 2.1fold $(\mathrm{p}<0.05)$ compared to undifferentiated group. The pronounced increase at $24 \mathrm{~h}$ was followed by a reduction to near basal expression with continued incubation for $48 \mathrm{~h}$. We also investigated the influence of NGF on the amount of Trk receptor protein in the cells. Figure 3B demonstrates that cells which were not exposed to NGF had considerable expression of TrkA, but the expression was more pronounced in NS- 1 which exhibited a 2 -fold increase in TrkA content relative to PC12. Treatment with NGF significantly increased the concentration of TrkA in NS-1 at $6 \mathrm{~h}$, remaining relatively constant or slightly reduced at $24 \mathrm{~h}$ and $48 \mathrm{~h}$.
PC12 cells on the other hand showed no change expression of TrkA under NGF differentiation at $6 \mathrm{~h}$ and $24 \mathrm{~h}$ compared to control. However, a 2 -fold $(\mathrm{p}<0.05)$ increase in the protein concentration of TrkA was detected in PC12 at $48 \mathrm{~h}$ post NGF exposure, but the increase was less than the basal expression in undifferentiated NS-1 cells.

\section{Discussion}

The use of in vitro cellular models has played a significant role in understanding the molecular and cellular processes relevant to disease pathogenesis and disease progression. PC12, a pheochromocytoma cell of adrenal medulla has proved a useful model system to study the mechanisms of neuronal growth, proliferation, differentiation, communication, injury and death for several decades. For several decades PC12 has proved a useful model system for studying neurodegenerative diseases including $\mathrm{AD}$ and Parkinson's [33,34]. However, this model has been confounding with several limitations that include time-consuming culture methods, protracted doubling time and long differentiation period. NS-1, a derivative cell line of PC12 has been gaining traction as an alternative model for phenotypic and molecular investigations that evaluate chemical effects on neurite outgrowth due to its enhanced sensitivity to NGF, accelerated doubling time, ease of handling, and inherent property to form elaborate neuritic processes in culture with NGF differentiation [2,5,30,35]. Differences in gene expression profile amongst diverse subclones of $\mathrm{SH}$ SY5Y neuroblastoma cells have been determined to play significant roles in modulating neurite outgrowth responses to NGF [36]. Given that both NS-1 and the parent cell line PC12 exhibit similar morphometric and phenotypic properties but on a much different time scale, we sort to investigate the differences in molecular architecture of the cells that could potentially account for these differences.

Trk receptors belong to a large family of membranespanning proteins that possess intrinsic kinase activity. Trks are essential for development and maintenance of the nervous system and control a large cascade of signaling processes that regulate cell survival, differentiation, proliferation, axonal elongation, neurite outgrowth, and synaptogenesis upon activation by growth factors [37-39]. The activation of Trk receptors by neurotrophins have been implicated in promoting survival and inducing apoptosis in PC12 [40,41]. Consequently, we assessed whether NS-1 cells express Trk receptors and compared the levels of expression to $\mathrm{PC} 12$ by gene expression analysis. Our results demonstrate that NS-1 expresses all three Trk receptor 
genes in undifferentiated state. Expression of Ntrk is crucial for the normal development and function of the nervous system. TrkA, the principal receptor for NGF plays important physiological role in the function of sympathetic neurons and is necessary for their survival and differentiation in vitro and in vivo [42]. We observed highly elevated transcription of Ntrkl and Ntrk2 in NS-1 cells following NGF differentiation, whereas the transcription in PC12 was unaltered. NGF is well-known to exert its neurotrophic actions via intracellular signaling mechanisms that lead to survival, proliferation and differentiation. The binding of TrkA receptor by NGF activates downstream signaling responses controlled by Ras/MAPK, phosphatidylinositol 3-kinase (PI3K)/Akt, and phospholipase C (PLC)- $\gamma$. Akt phosphorylation activates downstream genes to regulate neuronal survival either by inducing the transcription of survival genes or by inhibiting apoptosis. Overexpression study in PC-12 and SH-SY5Y suggest that NGF-induced increases in TrkA mRNAs expression is directly related to the formation of neurites [36,43]. Ntrk2 which encodes TrkB is upregulated in presence of BDNF and phosphorylates neural cell adhesion molecule (NCAM). However, TrkB can also interact with NCAM in absence of BDNF [44] and induces neurite outgrowth in hippocampal neurons. Given that NGF is the primary ligand of TrkA, our data imply that the marked change in Ntrkl gene expression in addition to Ntrk2 in NGFdifferentiated NS-1 suggests a role, to a certain extent, for the enhanced sensitization and rapid activation of differentiation program in NS-1 immediately after NGF treatment.

Muscarinic receptor signaling have been proposed to coordinate the response of neural networks in the CNS and thus, play an important role during brain development by regulating cell survival, proliferation, and differentiation. The stimulation of neuronal muscarinic receptors has been shown to induce neurite outgrowth in a variety of neuronal cell lines $[45,46]$ NGF differentiation of PC12 has also been previously reported to be accompanied by a large increase in the expression of muscarinic receptors [47]. Therefore, we investigated whether differences existed in the transcriptional regulation of $\mathrm{Chrm}$ gene family that could potentially indicate a role of these genes in phenotypic variations between NGF differentiated NS1 and PC12 cells. NGF differentiation increased the transcription of Chrm 2 in both cell lines. However, the regulation of Chrm 4 was inversely correlated in the cell lines relative to cells unexposed to NGF, with Chrm4 increasing greater than 3 -fold in PC12. NGF has been previously been reported to upregulate Chrm 2 receptor in $\mathrm{PC} 12$ potentially through inward rectifying potassium channel Kir3.2. In addition, NGF differentiated PC12 was shown to regulate $\mathrm{m} 4$ receptor mRNA stability through MAP kinase activity. Our findings are in agreement with these earlier reports on regulation of Chrm genes in PC12. The inverse correlation of $\mathrm{Chrm}$ gene transcripts in NS-1 point to complexities in the mechanisms that control transcription of Chrm genes in this cell line and could potentially be involved in surmounting the temporal constraints of NGF differentiation of NS-1 cells relative to the parental PC12. However, the role of these genes in the rapid changes in NS-1 subject to NGF treatment remains to be systematically investigated.

The adenosine receptor has been proposed to control NGF-induced neurite outgrowth in PC12 and primary hippocampal neuron [28]. Adora1 and Adora2A receptors which are present in neurons demonstrate opposing actions in the CNS and play significant roles in the regulation of neurotransmitters. In our study, we found that NS-1 cells differentially transcribed Adoral and Adora $2 A$ receptor mRNA in native state. These differences were normalized by NGF treatment. We also found that NGF-induced differentiation of NS-1 was accompanied by a highly significant downregulation of Adora1 gene transcription. The decrease in Adoral and Adora2A regulation observed in our study after treatment with NGF is consistent with previous research in PC-12 [48-50]. Adora1 receptor activation was shown to inhibit Rho-mediated formation of neurite outgrowth in PC12 [51]. Therefore, it is likely that the vast reduction in activity of Adoral gene ( 80 -fold) following NGF differentiation could have implication in the compressed timeline leading to rapid neuritogenesis, development of neurites, and formation of neuritic branch points by NS-1.

Acetylcholine (Ach), a neurotransmitter which promotes neuronal differentiation, is synthesized from choline and acetyl coenzyme A in a one-step reaction catalyzed by ChAT. Increase in ChAT mRNA is normally associated with differentiation of $\mathrm{PC} 12$ by NGF. Also, transfection of ChAT cDNA in the neuroblastoma cell N18TG2 lead to increased capacity of the cell to grow fibers, express synapsin 1, and induced neurites with carbachol treatment [46]. In our experiments we observed a 12 -fold increase ChAT mRNA in PC12 that was not seen in NS-1 after treatment with NGF. It is uncertain why the level of ChAT transcript was unaffected in NS-1 given prior observations in parental PC12. However, muted response in mRNA content of NS-1 could suggest a possible role for ChAT that is limited to cellular events 
without prime contribution to morphological changes associated with neurite outgrowth and elongation. This is unlikely because the protein content of ChAT was markedly upregulated in NS-1 after NGF exposure which indicates that expression of ChAT is controlled post-transcriptionally. Our data clearly suggests the operation of altered mechanisms for the control of ChAT expression in NS-1 and PC12 and warrants further enquiry.

PC12 cells synthesize and store catecholamines including dopamine and norepinephrine. They exhibit sympathetic neuronal properties and increase the expression of cholinergic markers, ChAT, and Ach activity in the presence of NGF [52-53]. NGF induces cholinergic differentiation by strongly elevating the expression of ChAT and increasing intracellular ACh levels through the PI3-kinase pathway [16]. In our study, we saw the first evidence of significant increase in ChAT protein expression in NS-1 at $6 \mathrm{~h}$ post NGF exposure. The increase was sustained for $24 \mathrm{~h}$ and was barely detectable at $48 \mathrm{~h}$. In contrast, a significant increase in ChAT protein was detected in PC12 at $24 \mathrm{~h}$ after NGF treatment. Unlike NS-1, ChAT protein expression was not sustained in PC12 but declined considerably at $48 \mathrm{~h}$, notwithstanding a 14 -fold increase in transcriptional activity of ChAT in PC12. Although we did not evaluate the protein levels of Ach in the cells, the early induction of ChAT expression in NS-1 following NGF exposure would predict a cognate increase in $\mathrm{ACh}$ as reported in the literature for PC12 and thus, drive early differentiation events in this cell model relative to PC12. ChAT is the main $\mathrm{ACh}$ synthesizing enzyme and its activity is strongly correlated and in balance to ACh levels. Enhanced secretion of ACh is essential for neurotransmission and may play a role in the activation of transcription factors such as early growth response protein 1 (EGR-1) essential for neurogenesis [54]. The existence of crosstalk has been established between several neurogenic pathways and studies have revealed association between TrkA and other cholinergic markers. Increased ChAT and VAChT mRNA have been observed in cells transfected with TrkA, while TrkA and ChAT proteins showed similar distribution pattern on the forebrain neurons during postnatal period. We observed peculiar differences in the expression of ChAT and VAChT in NS-1 and PC12. VAChT was expressed in PC12 and its regulation paralleled the expression of ChAT. In contrast, no evidence of VAChT expression was seen in differentiated NS-1 cells measured by immunoblot. The absence of VAChT in NS-1 is intriguing because it suggests the likelihood that distinct pathways exist for synthesis and secretion of ChAT in this cell line, similar to NG108-15 cells [55]. The inverse regulation between ChAT and VAChT at the gene and protein levels in NS1 is highly is thought-provoking and merits further studies as they could be potentially key determinants in the robust morphological and phenotypic characteristic exhibited by the cell line. Taken together our results imply that robust differences in NGF-dependent transcriptional controls of Trk, Chrm, Adora, and ChAT genes and associated translational regulation of Trk, ChAT, and VAChT protein expression potentially affect the responsive growth profiles, morphological attributes, and phenotypic properties of NS-1 and establishes NS-1 as a valid surrogate cell model for cholinergic neuronal research.

\section{Conclusion}

Drug discovery for modulators of neuronal phenotypes uses in vitro testing of compound libraries and complex imaging systems as primary screening tools. Therefore, the choice of cell line is critical when neurite outgrowth is the output criteria for analysis. PC12 cell line, the widely used model for neuronal differentiation studies presents numerous challenges in discovery and research environments due to prolonged culture and differentiation duration, growth requirements, and the tendency to form aggregates. Here we showed that NS1 cell, a derivative of $\mathrm{PC} 12$ exhibits enhanced characteristics relative to differentiation, growth and phenotypic properties in a shorter timeframe compared to the parental cell line. In addition, NS-1 cells express neuronal markers following differentiation with NGF. These characteristics support the emerging use of NS-1 as an alternate in vitro neuronal cell model.

\section{Acknowledgments}

Research reported in this publication was supported by National Institutes of General Medical Sciences of the National Institutes of Health under award number SC3GM116667. The content is solely the responsibility of the authors and does not necessarily represent the official views of the National Institutes of Health.

\section{Author Contributions}

G.I. and S.P. conceived and designed the experiments; C.H.L., S.P. and N.G. performed the experiments; S.P. and G.I. analyzed the data and wrote the paper.

\section{Conflicts of Interest}

The authors declare no conflict of interest. The funding sponsor had no role in the design of the study; in the collection, analyses, or interpretation of data; in the writing of the manuscript, and in the decision to publish the results. 


\section{References}

1. Das KP, Freudenrich TM, Mundy WR. Assessment of PC12 cell differentiation and neurite growth: a comparison of morphological and neurochemical measures. Neurotoxicol Teratol 2004; 26:397-406.

2. Radio NM, Breier JM, Shafer TJ, et al. Assessment of chemical effects on neurite outgrowth in PC12 cells using high content screening. Toxicol Sci 2008; 105:106-118.

3. Radio NM, Freudenrich TM, Robinette BL, et al. Comparison of PC12 and cerebellar granule cell cultures for evaluating neurite outgrowth using high content analysis. Neurotoxicol Teratol 2010; 32: 2535 .

4. Haas AJ, Le Page Y, Zhadobov M, et al. Effect of acute millimeter wave exposure on dopamine metabolism of NGF-treated PC12 cells. J Radiat Res 2017; 58: 439-445.

5. Yeyeodu ST, Witherspoon SM, Gilyazova N, et al. A rapid, inexpensive high throughput screen method for neurite outgrowth. Curr Chem Genomics 2010; 4: 74-83.

6. Greene LA, Tischler AS. Establishment of a noradrenergic clonal line of rat adrenal pheochromocytoma cells which respond to nerve growth factor. Proc Natl Acad Sci U S A 1976; 73: 2424-2428.

7. Scott SA, Mufson EJ, Weingartner JA, et al. Nerve growth factor in Alzheimer's disease: increased levels throughout the brain coupled with declines in nucleus basalis. J Neurosci 1995; 15: 6213-6221.

8. Tuszynski $\mathrm{MH}$, Thal L, Pay M, et al. A phase 1 clinical trial of nerve growth factor gene therapy for Alzheimer disease. Nat Med 2005; 11: 551-555.

9. Gall CM, Lauterborn JC, Guthrie KM, Stinis CT. Seizures and the regulation of neurotrophic factor expression: associations with structural plasticity in epilepsy. Adv Neurol. 1997; 72:9-24.

10. Hayakawa Y, Sakitani K, Konishi M, et al. Nerve Growth Factor Promotes Gastric Tumorigenesis through Aberrant Cholinergic Signaling. Cancer Cell 2017; 31: 21-34.

11. Dolle L, Adriaenssens E, El Yazidi-Belkoura I, et al. Nerve growth factor receptors and signaling in breast cancer. Curr Cancer Drug Targets 2004; 4: 463-470.

12. Wang W, Chen J, Guo X. The role of nerve growth factor and its receptors in tumorigenesis and cancer pain. Biosci Trends 2014; 8: 68-74.

13. Kim MS, Shutov LP, Gnanasekaran A, et al. Nerve growth factor (NGF) regulates activity of nuclear factor of activated T-cells (NFAT) in neurons via the phosphatidylinositol 3-kinase (PI3K)-Akt- glycogen synthase kinase 3beta (GSK3beta) pathway. J Biol Chem 2014; 289: 31349-31360.

14. Majdan M, Walsh GS, Aloyz R, et al. TrkA mediates developmental sympathetic neuron survival in vivo by silencing an ongoing p75NTRmediated death signal. J Cell Biol 2001; 155: 12751285.

15. Madziar B, Shah S, Brock M, et al. Nerve growth factor regulates the expression of the cholinergic locus and the high-affinity choline transporter via the Akt/PKB signaling pathway. J Neurochem 2008; 107: 1284-12893.

16. Madziar B, Lopez-Coviella I, Zemelko V, et al. Regulation of cholinergic gene expression by nerve growth factor depends on the phosphatidylinositol3'-kinase pathway. J Neurochem 2005; 92: 767-779.

17. Prado VF, Roy A, Kolisnyk B, et al. Regulation of cholinergic activity by the vesicular acetylcholine transporter. Biochem J 2013; 450: 265-274.

18. Chen KH, Reese EA, Kim HW, et al. Disturbed neurotransmitter transporter expression in Alzheimer's disease brain. J Alzheimers Dis 2011; 26: 755-766.

19. Ferreira-Vieira TH, Guimaraes IM, Silva FR, et al. Alzheimer's disease: Targeting the Cholinergic System. Curr Neuropharmacol 2016; 14: 101-115.

20. Galloway CR, Lebois EP, Shagarabi SL, et al. Effects of selective activation of M1 and M4 muscarinic receptors on object recognition memory performance in rats. Pharmacol 2014; 93: 57-64.

21. Leaderbrand $\mathrm{K}$, Chen HJ, Corcoran KA, et al. Muscarinic acetylcholine receptors act in synergy to facilitate learning and memory. Learn Mem 2016; 23: 631-638.

22. Fu Y, Tucciarone JM, Espinosa JS, et al. A cortical circuit for gain control by behavioral state. Cell 2014; 156: 1139-1152.

23. Foster DJ, Choi DL, Conn PJ, et al. Activation of M1 and M4 muscarinic receptors as potential treatments for Alzheimer's disease and schizophrenia. Neuropsychiatr Dis Treat 2014; 10: 183-191.

24. Xiang Z, Thompson AD, Jones CK, et al. Roles of the M1 muscarinic acetylcholine receptor subtype in the regulation of basal ganglia function and implications for the treatment of Parkinson's disease. J Pharmacol Exp Ther 2012; 340: 595-603.

25. Witkin JM, Overshiner C, Li X, et al. M1 and M2 muscarinic receptor subtypes regulate antidepressant-like effects of the rapidly acting antidepressant scopolamine. J Pharmacol Exp Ther 2014; 351: 448-456.

26. Simoes AP, Machado NJ, Goncalves N, et al. Adenosine A2A Receptors in the Amygdala Control 
Synaptic Plasticity and Contextual Fear Memory. Neuropsychopharmacol 2016; 41: 2862-2871.

27. Sheth S, Brito R, Mukherjea D, et al. Adenosine receptors: expression, function and regulation. Int $\mathbf{J}$ Mol Sci 2014; 15: 2024-2052.

28. Sun CN, Chuang HC, Wang JY, et al. The A2A adenosine receptor rescues neuritogenesis impaired by p53 blockage via KIF2A, a kinesin family member. Dev Neurobiol 2010; 70: 604-621.

29. Yeyeodu S, Gilyazova N, Huh EY, et al. A trifluoromethyl analog of verbenachalcone promotes neurite outgrowth and cell proliferation of NeuroScreen-1 cells. Cell Mol Neurobiol 2011; 31: 145-153.

30. Radio NM, Mundy WR. Developmental neurotoxicity testing in vitro: models for assessing chemical effects on neurite outgrowth. Neurotoxicol 2008; 29: 361-376.

31. Bradford MM. A rapid and sensitive method for the quantitation of microgram quantities of protein utilizing the principle of protein-dye binding. Anal Biochem 1976; 72: 248-254.

32. Pokharel S, Kamli MR, Mir BA, et al. Expression of Transthyretin during bovine myogenic satellite cell differentiation. In Vitro Cell Dev Biol Anim 2014; 50: 756-765.

33. Schlachetzki JC, Saliba SW, Oliveira AC. Studying neurodegenerative diseases in culture models. Rev Bras Psiquiatr 2013; 35 Suppl 2: S92-100.

34. Alberio T, Lopiano L, Fasano M. Cellular models to investigate biochemical pathways in Parkinson's disease. FEBS J 2012; 279: 1146-1155.

35. M KH, Kopp L, Kaur N, et al. A facile method for simultaneously measuring neuronal cell viability and neurite outgrowth. Curr Chem Genom Transl Med 2015; 9: 6-16.

36. Oe T, Sasayama T, Nagashima T, et al. Differences in gene expression profile among SH-SY5Y neuroblastoma subclones with different neurite outgrowth responses to nerve growth factor. J Neurochem 2005; 94: 1264-1276.

37. Vigneswara V, Kundi S, Ahmed Z. Receptor tyrosine kinases: molecular switches regulating CNS axon regeneration. J Signal Transduct 2012; 2012: 361721 .

38. Huang EJ, Reichardt LF. Trk receptors: roles in neuronal signal transduction. Annu Rev Biochem 2003; 72: 609-642.

39. Luikart BW, Parada LF. Receptor tyrosine kinase Bmediated excitatory synaptogenesis. Prog Brain Res 2006; 157: 15-24.

40. Loeb DM, Greene LA. Transfection with Trk restores "slow" NGF binding, efficient NGF uptake, and multiple NGF responses to NGF-nonresponsive PC1 2 cell mutants. J Neurosci 1993; 13: 2919-2929.

41. Ioannou MS, Fahnestock M. ProNGF but Not NGF, Switches from Neurotrophic to Apoptotic Activity in Response to Reductions in TrkA Receptor Levels. Int J Mol Sci 2017; 18.

42. Fagan AM, Zhang H, Landis S, et al. TrkA, but not TrkC, receptors are essential for survival of sympathetic neurons in vivo. J Neurosci 1996; 16: 6208-6218.

43. Hempstead BL, Rabin SJ, Kaplan L, et al. Overexpression of the Trk tyrosine kinase rapidly accelerates nerve growth factor-induced differentiation. Neuron 1992; 9: 883-896.

44. Cassens C, Kleene R, Xiao MF, et al. Binding of the receptor tyrosine kinase TrkB to the neural cell adhesion molecule (NCAM) regulates phosphorylation of NCAM and NCAM-dependent neurite outgrowth. J Biol Chem 2010; 285: 2895928967.

45. Tata AM, Cursi S, Biagioni S, et al. Cholinergic modulation of neurofilament expression and neurite outgrowth in chick sensory neurons. J Neurosci Res 2003; 73: 227-234.

46. De Jaco A, Augusti-Tocco G, Biagioni S. Muscarinic acetylcholine receptors induce neurite outgrowth and activate the synapsin I gene promoter in neuroblastoma clones. Neurosci 2002; 113: 331338.

47. Jumblatt JE, Tischler AS. Regulation of muscarinic ligand binding sites by nerve growth factor in PC12 phaeochromocytoma cells. Nature 1982; 297: 152154.

48. Lee $\mathrm{NH}$, Weinstock $\mathrm{KG}$, Kirkness EF, et al. Comparative expressed-sequence-tag analysis of differential gene expression profiles in PC-12 cells before and after nerve growth factor treatment. Proc Natl Acad Sci U S A 1995; 92: 8303-8307.

49. Arslan G, Kontny E, Fredholm BB. Downregulation of adenosine $\mathrm{A} 2 \mathrm{~A}$ receptors upon NGFinduced differentiation of PC12 cells. Neuropharmacol 1997; 36: 1319-1326.

50. Nie Z, Mei Y, Malek RL, et al. A role of p75 in NGF-mediated down-regulation of the $\mathrm{A}(2 \mathrm{~A})$ adenosine receptors in PC12 cells. Mol Pharmacol 1999; 56: 947-954.

51. Thevananther S, Rivera A, Rivkees SA. A1 adenosine receptor activation inhibits neurite process formation by Rho kinase-mediated pathways. Neuroreport 2001; 12: 3057-3063.

52. Baizer L, Weiner N. Nerve growth factor treatment enhances nicotine-stimulated dopamine release and increases in cyclic adenosine $3^{\prime}: 5^{\prime}$-monophosphate 
levels in PC12 cell cultures. J Neurosci 1985; 5: 1176-1179.

53. Avila AM, Davila-Garcia MI, Ascarrunz VS, et al. Differential regulation of nicotinic acetylcholine receptors in $\mathrm{PC} 12$ cells by nicotine and nerve growth factor. Mol Pharmacol 2003; 64: 974-986.

54. Anelli $\mathrm{T}$, Cardarelli $\mathrm{S}$, Ori $\mathrm{M}$, et al. 5Hydroxytryptamine $1 \mathrm{~A}$ and $2 \mathrm{~B}$ serotonin receptors in neurite outgrowth: involvement of early growth response protein 1. Dev Neurosci 2013; 35: 450460.

55. Castell X, Cheviron N, Barnier JV, et al. Exploring the regulation of the expression of ChAT and VAChT genes in NG108-15 cells: implication of PKA and PI3K signaling pathways. Neurochem Res 2003; 28: 557-564.

This manuscript was peer-reviewed

Mode of Review: Single-blinded

Academic Editor: Dr. Jose Ricardo Kina and Dr. Li Pin Kao

Copyright: (92018 Pokharel S, et al. This article is distributed under the terms of the Creative Commons Attribution 4.0 International License [http://creativecommons.org/licenses/by/4.0/], which permits unrestricted use, distribution, and reproduction in any medium, provided you give appropriate credit to the original author[s] and the source, provide a link to the Creative Commons license, and indicate if changes were made. 